\title{
Les écoulements autour des ailes ventilées
}

\section{Jacques Dodu}

Professeur

\section{Jean-Marie Michel}

Chargé de Recherche au C.N.R.S.

\section{Alain Rowe}

Ingénieur au C.N.R.S.

Institut de Mécanique de Grenoble

L'étude des écoulements autour des ailes cavitantes ou ventilées présente un intérêt technique considérable puisque son champ d'application concerne tous les mouvements relatifs rapides de profils ou d'obstacles au sein d'un liquide. On peut citer à titre d'exemple les ailes sous-marines et les pales d'hélices des navires rapides, les pompes supercavitantes, les entrées d'eau. Les études dont nous souhaitons exposer les résultats concernent des ailes portantes à cavitation de culot-ou cavitantes à la base- dont l'extrados mouillé permet d'escompter que la finesse ne s'abaisse pas au-dessous de 9 en écoulement tridimensionnel et de 12 ou 14 en configuration bidimensionnelle. La pression dans la cavité peut résulter de la présence de la vapeur seule ou de vapeur et d'air entrainé ou injecté. Dans les deux cas, la pression de cavité constitue l'un des paramètres essentiels du phénomène et l'hydrodynamique globale ne dépend pas d'une manière appréciable de la nature du gaz de la cavité, comme on peut aisément le vérifier par l'expérience. Techniquement, la ventilation retient l'attention par son influence prépondérante sur la traînée qu'elle réduit par l'augmentation de la pression de culot. L'utilisation des ailes ventilées tronquées se justifie par le fait que la répartition judicieuse des pressions sur l'extrados encore mouillé limite à des valeurs acceptables la diminution de portance que ne manquerait pas d'engendrer l'augmentation de pression dans la cavité. Bien entendu, les conditions de fonctionnement des ailes impliquent qu'on veille soigneusement à éviter toute cavitation de bord d'attaque.

Lorsque les ailes circulent à très grande vitesse, les couches limites qui s'établissent sur les parois sont très minces et dans leur environnement proche la répartition des pressions locales ne s'écarte guère de celle du champ irrotationnel associé aux frontières effectives du profil. $\mathrm{Si}$ de plus l'aile et sa cavité forment un ensemble très allongé dans la direction privilégiée du déplacement (généralement la direction horizontale) on est fondé à penser que toute modélisation respectant au moins la géométrie de l'aile permet d'accéder sans trop d'erreur aux propriétés globales -portance, trainée, momentdont la connaissance est nécessaire au constructeur. En écoulement bidimensionnel, cette idée se trouve à la base de développements qui ont connu jusqu'à un passé proche un succès considérable [1] à [3] et il est commode de l'évoquer comme point de départ de recherches qui ont fait l'objet de travaux théoriques et expérimentaux récents.

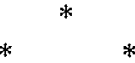

De fait, l'observation expérimentale sur ce sujet joue un rôle assez déterminant et sa pratique inspire les principales remarques qui suivent.

D'abord, la conduite d'essais de cavitation requiert des moyens importants (dimensions des tunnels ou des canaux, puissances mises en jeu) et assez performants. A l'Institut de Mécanique de Grenoble un effort particulier a été accompli [4] de manière qu'on puisse atteindre des nombres de cavitation $\sigma$ et des dépressions relatives de cavités $\sigma$ assez petits $(0,02$ et 0,015 respectivement) soit en cavitation de vapeur, soit par ventilation ; dans ce dernier cas, on a dû prévoir un dégazage énergique à l'aval de la veine de mesure du tunnel qui fonctionne en circuit fermé.

On remarque que les résultats expérimentaux relatifs à la géométrie des cavités et à la dynamique des profils fournissent pour les modélisations analytiques les éléments de contrôle indispensables et des données intermédiaires. Par exemple, la longueur de cavité joue en écoulement bidimensionnel un róle très significatif comme donnée intermédiaire. C'est une longueur effectivement accessible par l'expérience moyennant quelques précautions $[5, \mathrm{III}]$; on sait qu'elle dépend strictement de la dépression relative de la cavité et la logique conduit à la considérer comme paramètre d'entrée de calcul, 
associée à la géométrie du profil pour aboutir en fait à la détermination de $\sigma$. Enfin, élément essentiel de l'aspect hydrodynamique de l'écoulement, elle évolue d'une manière continue dans tous les cas en fonction de la dépression relative de la cavité alors que, dans le cas des écoulements à cavités pulsantes, celle-ci varie d'une manière discontinue en fonction du débit d'air, paramètre essentiel de la ventilation.

En l'état actuel, les lacunes de la connaissance qu'on a sur les mécanismes qui déterminent la fermeture des cavités et par conséquent le taux d'air entrainé rendent sans doute opportun d'approfondir la recherche expérimentale qui les concernent. Il ne s'agit pas d'effectuer seulement une tentative en vue de satisfaire une curiosité scientifique légitime, mais en méme temps de répondre aux préoccupations techniques des maitres d'œuvre et des constructeurs. La zone de fermeture constitue en effet un domaine où plusieurs phénomènes se produisent d'une manière simultanée dans le temps et imbriquée dans l'espace : condensation de vapeur, entrainement d'air par bulles ou par poches, dissipation d'énergie due soit au développement des couches limites nées sur les parois des profils, soit aux conséquences de l'instabilité propre de l'écoulement limité par les surfaces libres de la cavité. En outre, dans le cas des écoulements tridimensionnels, l'apparition d'une cavité de bout d'aile, liée à l'évolution de la circulation en envergure, constitue une complication supplémentaire qui rend difficile la modélisation et risque d'en compromettre le succès en l'absence de relevés géométriques issus de l'expérience.

$$
* * *
$$

Quelles qu'en soient les difficultés et l'imperfection, la modélisation analy tique permet d'obtenir des résultats de grand intérêt.

Pour l'étude des écoulements bidimensionnels, on profite naturellement de l'efficacité d'emploi de la transformation conforme, qui permet d'obtenir avec précision la solution des problèmes aux frontières. Mais pour ces écoulements, il y a lieu de distinguer d'emblée à côté de la procédure de traitement exacte la procédure de linéarisation. Grâce à la linéarisation, le problème intégral incluant la recherche de la frontière inconnue de la cavité se ramène à un problème sur une frontière définie. Comme pour l'aérodynamique des ailes minces, c'est la nature même des profils qui justifie l'emploi de la linéarisation. En effet ces profils sont peu épais, peu cambrés et peu inclinés par rapport à la direction générale du mouvement. De plus, si la vitesse est grande, la cavité est mince et longue et les insuffisances de la schématisation n'apparaissent qu'au bord d'attaque, à la fermeture de la cavité et éventuellement dans le sillage.

Dans ce cadre, deux tentatives pour améliorer les résultats disponibles ont été menées à bien :

- L'emploi de la procédure des développements asymptotiques raccordés [6] permet de résoudre d'une manière satisfaisante le calcul des coefficients de pression locaux au bord d'attaque et de déterminer notamment la fourchette des incidences de noncavitation dans cette région.
- La modélisation des sillages, avec prise en compte de la pression effective dans le sillage, ou d'une manière un peu équivalente du défaut de quantité de mouvement dú à la présence du profil et de sa cavité dans le champ initialement uniforme [7], permet de calculer une configuration d'écoulement et des propriétés hydrodynamiques plus proches de celles qu'on obtient par l'expérience.

$$
* * *
$$

L'exposé qui suit montre que les résultats de ces recherches conduisent à une vision plus élaborée des écoulements rapides autour de profils minces, avec des conclusions transposables au comportement des ailes réelles tridimensionnelles.

En fait, pour ces profils, la possibilité de linéariser conduit à assimiler l'élément hydrodynamique essentiel, la portance, à un terme de perturbation. Du point de vue physique, il revient au même de constater que la déviation globale de l'écoulement par le profil perpendiculairement à la direction du déplacement est petite. Dans ces conditions, on doit s'attendre à une influence non négligeable de toute autre perturbation susceptible de modifier la répartition des quantités de mouvement transversales. Au voisinage du profil, cette influence se fera surtout sentir par la modification du point d'arrêt au bord d'attaque, dans une région où la valeur des coefficients de pression locaux est très importante et leur répartition déterminante. On décrit aussi le méme phénomène en disant que, sous l'influence d'une telle perturbation, l'incidence d'adaptation du profil est modifiée : perturber la déviation transversale déplace le point d'arrêt, auquel on peut redonner sa position antérieure en modifiant convenablement l'incidence du profil.

Dans les conditions habituelles de fonctionnement des profils portants en écoulement rapide, on reconnait facilement plusieurs causes principales de déviation globale de l'écoulement, toutes marquées par la dissymétrie par rapport à la direction générale du déplacement : - présence d'une surface libre, avec influence de la profondeur d'immersion,

- présence d'une surface libre et d'un fond, présence du champ de gravité,

- envergure limitée de l'aile.

Les résultats suivants, présentés d'une manière détaillée, sont particulièrement significatif à cet égard.

\section{Paramètres descriptif́s de l'écoulement, longueur des cavités}

De la description physique des écoulements plans à cavités de vapeur ou à cavités ventilées, déjà présentée dans un article de La Houille Blanche [5, III] nous ne retenons ici que les points essentiels, notamment ceux qui concernent les paramètres déterminants de l'écoulement et l'évolution des longueurs de cavité.

En se référant au cas simplifié de la figure 1 , on dénombre comme paramètres primaires : la corde $c$ du 


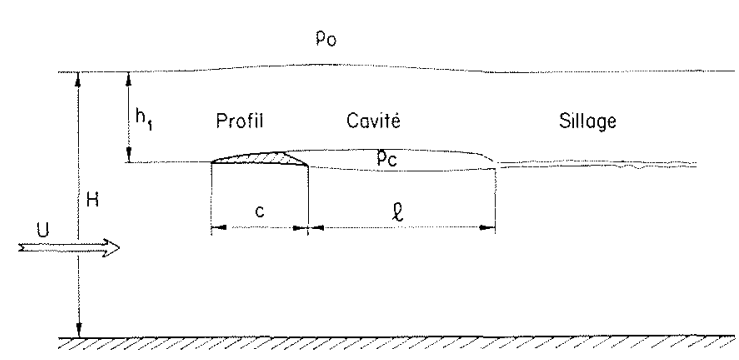

Figure 1 - Schéma de l'écoulement en canal.

profil, la profondeur d'immersion $h_{1}$, l'incidence $\alpha$, la profondeur du canal $H$, la pression $p_{o}$ à la surface libre, la vitesse $U$ de l'eau à l'entrée du canal et éventuellement le débit-masse d'air injecté $Q$; enfin l'intensité de la pesanteur et les propriétés physiques des fluides en présence, principalement de l'eau : masse volumique $\rho$, tension de vapeur $p_{v}$, viscosité, tension superficielle. La longueur $\ell$ de la cavité, la pression $p_{c}$ dans la cavité et les efforts subis par le profil constituent les grandeurs dépendantes essentielles.

Dans le cas des cavités naturelles, l'expérience montre que la pression de cavité diffère très peu de la tension de vapeur en raison de la très faible diffusion de l'air dissous du liquide vers la cavité lorsque celle-ci est pleinement développée. Dans le cas des cavités ventilées, on admet que la cavité est saturée en vapeur :

$$
p_{c}=p_{v}+P_{\mathrm{air}}
$$

L'occurence éventuelle de phénomènes périodiques conduit à retenir d'abord pour l'analyse les valeurs moyennes des pressions $\bar{p}_{c}, \bar{P}_{\text {air }}$ et aussi de la longueur de cavité $\bar{\ell}$.

La dépression relative constitue le paramètre hydrodynamique essentiel puisqu'elle est à la base de la prévision des caractéristiques globales de l'écoulement : efforts globaux, longueur de cavité :

$$
\sigma=\Delta \bar{p} / \frac{1}{2} \rho U^{2}=\left[p_{o}+\rho g h_{1}-\bar{p}_{c}\right] / \frac{1}{2} \rho U^{2} .
$$

En prenant pour référence la pression hydrostatique au niveau du profil on espère pouvoir comparer efficacement les résultats expérimentaux et les résultats théoriques obtenus assez généralement à partir de schémas qui ne tiennent compte que de l'inertie du liquide et qui négligent l'influence de la gravité.

Dans le cas des cavités de vapeur, la dépression relative de la cavité $\sigma$ s'identifie au nombre de cavitation de Thoma $\sigma_{v}$ qui fixe les conditions globales de l'écoulement, au contraire, dans le cas des cavités ventilées, la dépression relative qui s'écrit :

$$
\sigma=\sigma_{v}-\sigma_{a} \text { avec } \sigma_{a}=\bar{P}_{\text {air }} / \frac{1}{2} \rho U^{2}
$$

est un des éléments de la réponse de l'écoulement à toutes les grandeurs d'entrée : la valeur de la pression de l'air dans la cavité dépend du mode d'entraînement de l'air à l'aval de la cavité et donc de toute la dynamique de l'écoulement. Il est alors essentiel de préciser la dépendance de grandeurs telles que la pression $\bar{P}_{\text {air }}$ et la longueur $\bar{l}$ par rapport à l'ensemble des grandeurs d'entrée. A cet égard la méthode de l'analyse dimensionnelle fournit au moins un cadre de travail : nous en indiquons ci-dessous les conclusions et nous exposons les premiers résultats obtenus.

En définissant le nombre de cavitation avec pour référence la pression hydrostatique

$$
\left(\sigma_{v}=\left[p_{o}+\rho g h_{1}-p_{v}\right] / \frac{1}{2} \rho U^{2}\right)
$$

on obtient pour la pression d'air :

$$
\sigma_{a}=\sigma_{a}\left[\frac{H}{c}, \frac{h_{1}}{c}, \alpha, \sigma_{v}, C_{Q_{m}} \text { ou } C_{Q_{c}}, F r, W, R e\right]
$$

et de la même manière pour la dépression relative :

$$
\sigma=\sigma\left[\frac{H}{c}: \frac{h_{1}}{c}, \alpha, \sigma_{\nu}, C_{Q_{m}} \text { ou } C_{Q_{c}}, F r, W, R e\right]
$$

Dans ces relations $C_{Q_{m}}$ désigne le coefficient de débit-masse d'air injecté, $C_{Q_{c}}$ le coefficient de débitvolume d'air a la pression de l'air dans la cavité, coefficient que l'on peut considérer comme une évaluation du rapport de la vitesse de l'air dans la cavité à la vitesse de l'eau; les quantités $F r, W, R e$ désignent respectivement les nombres de Froude, de Weber et de Reynolds. La dépendance par rapport à l'incidence signifie que l'écoulement de l'air à l'arrière de la cavité est déterminé partiellement par la circulation autour du profil. Enfin la présence des grandeurs $H / c$ et $h_{1} / c$ traduit l'influence des frontières extérieures de l'écoulement : l'immersion relative $h_{1} / c$ est plus particulièrement significative.

L'expérience et l'analyse directe montrent que la géométrie moyenne de l'écoulement est fixée principalement par la dépression relative indépendamment de la manière dont la pression de cavité est établie :

$$
\frac{\bar{\ell}}{c}=\frac{\bar{\ell}}{c}\left[\frac{H}{c}, \frac{h_{1}}{c}, \alpha, \sigma, F r, W, R e\right] .
$$

Les coefficients d'efforts globaux s'expriment par des relations de dépendance analogues à la relation (c) puisque la portance et la traînée sont déterminées par la géométrie de l'écoulement.

L'étude de l'évolution de la longueur moyenne de cavité en fonction de la dépression relative se révèle intéressante parce qu'elle permet par des mesures assez simples de longueurs, de vitesses et de différences de pression de comparer la théorie et l'expérience. Pour un profit tenu à incidence nulle et pour des vitesses rendant négligeables les forces de gravité, on obtient une loi du type

$$
\frac{\bar{\ell}}{c}=A\left(\frac{\epsilon}{\sigma}\right)^{n}
$$

où $\epsilon$ désigne un petit paramètre caractéristique de l'épaisseur relative du profil et où le coefficient $A$ et 
l'exposant $n$ dépendent surtout de l'immersion relative $h_{1} / c: n$ croit avec $h_{1} / c$; pour $h_{1} / c<1, n \simeq 1$; pour: $h_{1} / c \gg 1, n \simeq 2$. L'augmentation de l'incidence se traduit par une diminution de la longueur de cavité. Dans les conditions des expériences, les dépressions relatives sont comprises entre 0,02 et 0,2 environ, ce qui peut correspondre pour un profil assez épais ( $\epsilon$ voisin de $0,28)$ à des longueurs de cavités comprises entre 20 et 2. Dans le troisième paragraphe de cet article, on montrera que la loi expérimentale $\left(c^{\prime}\right)$ est assez bien retrouvée à partir d'une formulation théorique basée sur le schéma de l'écoulement à potentiel de vitesse, au moins en ce qui concerne la valeur de l'exposant $n$.

La dépendance de la dépression relative de la cavité à l'egard des divers paramètres qui figurent dans la relation (b) apparaît d'emblée comme beaucoup plus compliquée à élucider. D'une part, le phénomène de pulsation des cavités se traduit par des discontinuités du paramètre $\sigma$ (et corrélativement de la longueur des cavités et des efforts globaux sur le profil), lors des changements de régime de pulsation, et cela pour des variations continues du débit d'air ou de l'incidence ; si les mécanismes de pulsation et de changement de régime sont connus au moins qualitativement [5, III], il est actuellement impossible de prédire les valeursseuils du débit d'air ou de l'incidence pour lesquelles la géométrie de l'écoulement est modifiée. Il est notable aussi que ces valeurs-seuils diffèrent suivant que l'on augmente ou que l'on diminue le débit d'air ou l'incidence, donnant par là naissance à des cycles d'hystérésis. D'autre part, en l'absence même du phénomène de pulsation des cavités - l'écoulement de l'air à l'aval des cavités étant alors continu ou quasi continu - l'expérience montre que, pour une géométrie donnée du profil dans le canal, la relation entre le débit d'air et le nombre $\sigma$ fait apparaitre une forte dépendance à l'égard de la vitesse $U$ :

$$
\sigma=\sigma\left[\sigma_{v}, C_{Q_{c}}, \Pi(U)\right]
$$

sans que l'on connaisse les mécanismes impliqués dans cette dépendance. Comparant les relations $\left(b^{\prime}\right)$ et (b), on est tenté d'attribuer la dépendance à l'égard de la vitesse aux forces de viscosité, de tension superficielle ou de gravité : ces forces contribuent à déterminer la microgéométrie des frontières de la cavité, grandeur que 1 on soupçonne d'influer partiellement sur les conditions de fermeture de la cavité et par là même sur le taux d'entraînement d'air à l'aval. S'il en est effectivement ainsi, toute autre cause de perturbation des frontières de la cavité - par exemple la turbulence préexistante de l'écoulement et la rugosité du profil doit être prise en compte aussi dans l'analyse de la relation entre le débit d'air et la pression dans la cavité : des essais ponctuels tendent effectivement à affermir cette opinion.

Le caractère tridimensionnel des écoulements autour d'ailes réelles d'envergure limitée influe considérablement sur la formation des cavités et leur pulsation éventuelle. La pulsation des cavités ventilées est liée surtout à laspect bi-dimensionnel des écoulements : dans ce type d'ecoulement, la cavité tend à être fermée par un jet rentrant qui bloque l'air à l'intérieur et interdit son échappement continu vers l'aval. Toute perturbation de type tridimensionnel qui tend à détruire la structure de jet rentrant ou qui permet à l'air de contourner le blocage dû au jet rentrant, tend aussi à détruire le phénomène de pulsation. Les perturbations peuvent être locales, comme celles qui sont dues à la géométrie fine des frontières de la cavité : dans ce cas, qui se produit même si l'écoulement est globalement à deux dimensions, la structure de jet rentrant est plus facilement détruite si l'épaisseur relative de la cavité est plus petite. Les perturbations peuvent être aussi globales comme dans le cas d'une aile d'envergure finie où, à côté de la cavité centrale de type bidimensionnel, se développe une cavité marginale liée au tourbillon d'extrémité dans laquelle l'air tend à s'écouler de manière préférentielle. L'expérience a cependant montré que la pulsation des cavités peut se produire dans le cas de demi-ailes d'allongement 2, pour des profils ayant, il est vrai, une épaisseur relative assez grande. La présence de deux cavités et la possibilité de la pulsation des cavités fait apparaitre une grande variété de configurations des écoulements tri-dimensionnels qui rend délicate la recherche des domaines de valeurs des paramètres qui les déterminent.

\section{Quelques caractéristiques hydrodynamiques essentielles des ailes ventilées}

C'est en 1972 que l'intéraction considérable qui existe entre la cavité et le profil (schématisé sur la figure 1) lorsque ce dernier se trouve placé à proximité d'une surface libre, a été mise pour la première fois en évidence $[5, \mathrm{II}]$. Cette intéraction justifie l'effort entrepris en vue d'approfondir les connaissances concernant la ventilation des cavités développées, et principalement la relation pression-longueur de cavité. Les variations de la longueur de la cavité sous l'effet d'une modification du soufflage ou éventuellement de la vitesse s'accompagnent en effet d'une déformation de la surface libre, frontière de l'écoulement, déformation qui se traduit pour le profil par l'apparition d'une incidence induite $\Delta \alpha_{i}$. Cette incidence induite a pour conséquence de faire apparaitre un minimum sur la courbe d'évolution du coefficient de portance $C_{z}$ en fonction de la dépression relative $\sigma$.

En pratique, on constate qu'une augmentation de la longueur de cavité a pour effet de faire augmenter la portance du profil lorsque la longueur de la cavité est au moins de l'ordre d'une corde (la portance diminue si $\ell<1$ corde). Cette augmentation de la portance, à incidence géométrique fixée, s'accompagne d'une diminution de l'incidence d'adaptation du profil. Un calcul effectué en théorie linéarisée, corrigé au bord d'attaque par la méthode des développements asymptotiques raccordés [6] a confirmé un point important par ailleurs prévisible intuitivement, à savoir qu'à l'incidence d'adaptation la portance du profil reste pratiquement constante. Cette dernière assertion reste d'ailleurs vraie pour toute déformation géométrique du champ de l'écoulement, et notamment pour une variation de la profondeur d'immersion du profil. Le même calcul a permis d'obtenir les valeurs des coefficients d'efforts pour des conditions géométriques variées (profondeur d'immersion, 


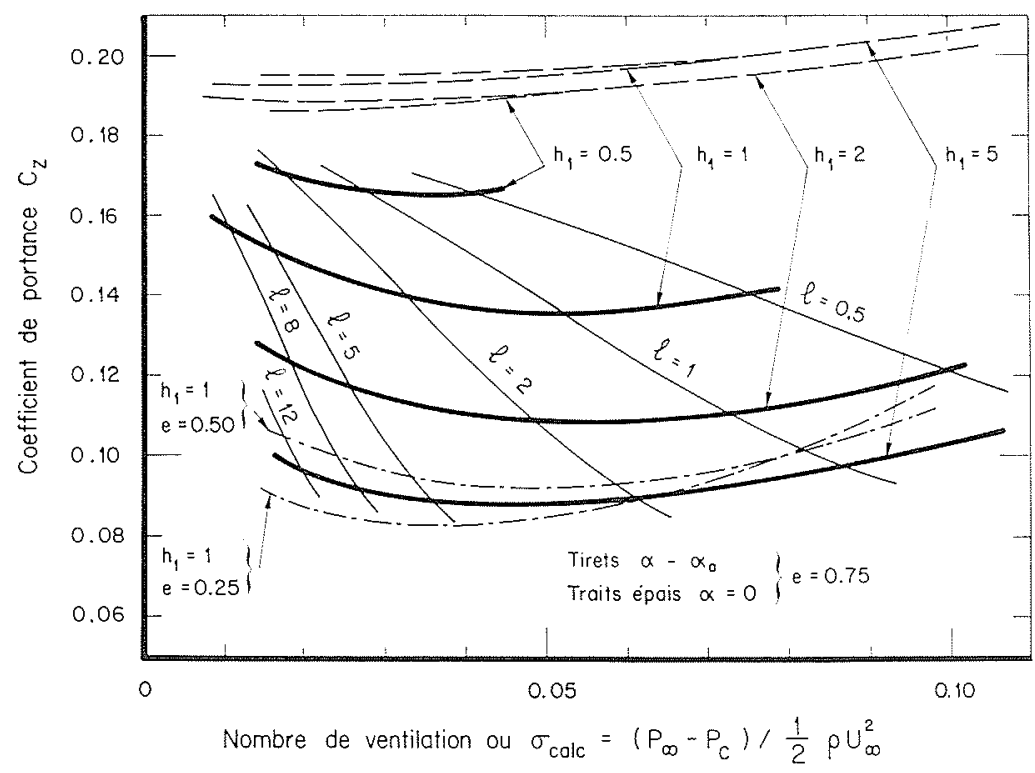

Figure $2-C_{z}(\sigma)$ théorique $[6]$.

longueur de la cavité, incidence), ainsi que l'étendue du domaine de non-cavitation (fig. 2 et 3 ). Compte tenu de l'importance de l'intéraction "cavité-profil" en présence d'une surface libre, il y avait lieu de prévoir que les résultats théoriques obtenus à partir d'un modèle représentant l'écoulement, et en particulier la cavité et le sillage, ne seraient tout-à-fait acceptables que pour autant que ce modèle soit apte à restituer une loi de correspondance correcte $\ell(\sigma)$. En ce qui concerne les calculs évoqués ci-dessus, la trop grande schématisation de la région du sillage a malheureusement pour effet de restituer une longueur de cavité trop courte pour un nombre de ventilation donné, ce qui se traduit par une mauvaise appréciation de l'incidence induite $\Delta \alpha_{i}$, d'où résulte pour la portance un décalage entre les valeurs trouvées expérimentalement et les

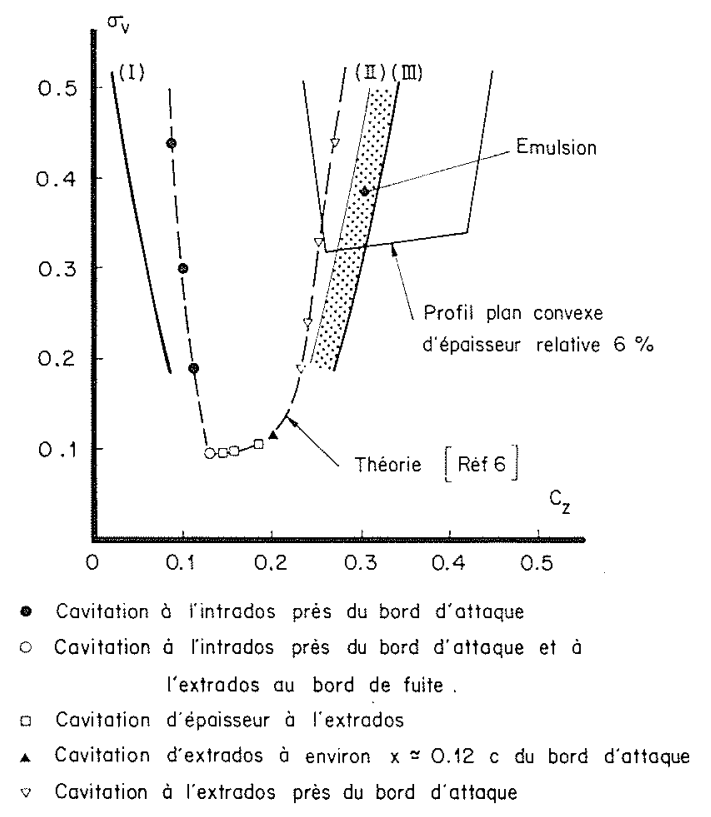

Figure 3 - Limite de cavitation pour un profil d'épaisseur relative équivalente (mesurée à la base) égale à $12 \%$. valeurs calculées. Il est cependant agréable de constater que le modele utilisé, bien qu'imparfait, permet d'obtenir pour la portance le comportement qualitatif décrit précédemment en confirmant l'existence du minimum (fig. 2). Pour améliorer la correspondance quantitative entre les résultats théoriques et expérimentaux, il est nécessaire d'utiliser un modèle représentatif de l'écoulement plus élaboré, principalement au niveau du sillage. Un essai a été effectué dans ce sens, qui fait l'objet du paragraphe suivant.

L'étude du domaine de non-cavitation fait également ressortir une bonne concordance entre les résultats théoriques et expérimentaux. Sur la figure 3, nous avons représenté les limites de cavitation relatives au même profil, obtenues théoriquement et expérimentalement. Les courbes (I), (II) et (III) représentent les limites de cavitation de bord d'attaque, relevées expérimentalement à l'intrados et à l'extrados. A l'extrados nous avons tracé deux courbes, car la cavitation apparait d'abord sous forme d'une émulsion très ténue (courbe (II)), qui s'épaissit puis se concentre au bord d'attaque (courbe (III)). La bonne concordance avec les résultats théoriques (courbe en tirets) s'explique par le fait que le domaine d'utilisation est paramétré en coefficients de portance, et non en angles d'incidence. Comme la fourchette de non-cavitation est étroite, le profil se trouve toujours placé au voisinage de l'incidence d'adaptation ; or nos résultats montrent qu'à l'incidence d'adaptation la portance prévue par le calcul est correcte, étant entendu que le calage du profil pour lequel cette portance est obtenue est entaché d'une erreur. Cette assertion s'appuie sur le résultat théorique suivant : le calage théorique correspondant au milieu de la fourchette de non-cavitation coinncide à très peu près avec le calage théorique correspondant à l'incidence d'adaptation. Cela confère d'ailleurs au problème du pilotage d'une aile réelle un caractère de sévérité moindre, puisque le pilotage se faisant en principe à portance constante, on est assuré que le profil reste dans tous les cas calé au milieu de la fourchette de non-cavitation. On voit sur la figure 3 que les résul- 
tats théoriques paraissent un peu pessimistes par rapport aux résultats expérimentaux. L'écart observé ne parait pas excessif si l'on se réfère à d'autres études similaires. Sur la figure 3 , nous avons enfin fait figurer les limites de cavitation correspondant à un profil plan convexe de $6 \%$ d'épaisseur relative [10]. Un tel profil simule assez bien un profil plus évolué de type NACA 16 par exemple pour lequel la limite de cavitation d'épaisseur serait vraisemblablement très semblable. La comparaison avec les limites de cavitation du profil ventilé fait apparaitre la nette supériorité de ce dernier pour l'obtention des grandes vitesses.

\section{Modélisation du sillage de la covitité [7]}

Ce paragraphe est consacré à l'exposé d'une méthode de calcul, en théorie linéarisée, de l'écoulement considéré dans la figure 1, une attention particulière étant apportée aux conditions aux limites sur le sillage de la cavité et à leur influence sur la prévision du comportement de l'écoulement.

On cherche à déterminer la vitesse complexe de perturbation $w=u-i v$ en fonction de la variable complexe $z=x+i y$ du plan physique linéarisé dont les frontières sont constituées par des segments rectilignes (ici les longueurs sont rapportées à la corde de l'intrados du profil prise pour unité). Les conditions aux limites, reportées sur la figure 4, se déduisent soit de l'équation de Bernoulli dans le cas des surfaces libres, soit de la condition de glissement sur les parois solides. En particulier, sur le profil d'équation $y=\epsilon F(x)$, où $\epsilon$ désigne le petit paramètre qui caractérise l'épaisseur du profil, la condition de glissement impose la valeur de la composante transversale $v$ de la vitesse de perturbation.

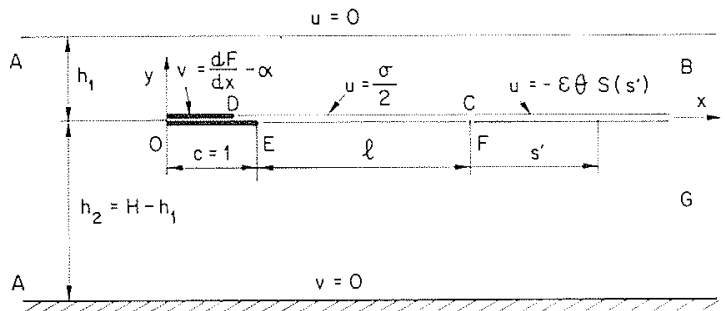

Figure 4 - Plan physique linéarisé. Longueur de l'extrados: $e$.

Les conditions sur le sillage de la cavité revêtent un certain arbitraire puisqu'il est impossible de rendre compte analytiquement de la complexité des phénomènes dont le sillage est le siège. Dans le schéma habituellement utilisé selon lequel ces conditions auraient peu d'influence sur le comportement du profil, au moins lorsque la cavité est assez longue, on impose seulement une survitesse longitudinale nulle : $u=0$ sur le sillage de la cavité (modèle appelé $S_{0}$ ). Cependant, de cette condition, résulte une relation $\ell(\sigma)$ incorrecte [6] : si l'on retrouve à peu près les valeurs de l'exposant $n$ de la loi expérimentale $\left(c^{\prime}\right)$, le coefficient $A$ théorique est environ 2,4 fois trop petit. Puisque, pour une dépression relative de la cavité fixée, la géométrie de l'écoulement n'est pas correcte, on doit s'attendre à ce que l'incidence d'adaptation du profil ne soit pas convenablement calée et que le coefficient de portance théorique soit entaché d'erreur. Signalons que ces difficultés n'apparaissent pratiquement pas si l'on considère un milieu fluide infini ou limité par des parois solides. Il est donc important, d'une part, d'effectuer les expériences avec un moyen d'essai qui restitue les conditions de surface libre que le profil doit rencontrer dans la réalité ; d'autre part, il est nécessaire, dans une étude théorique qui prend en compte la surface libre extérieure de l'écoulement, d'améliorer les conditions aux limites sur le sillage de la cavité.

Les conditions qui ont été choisies tentent de simuler le défaut de quantité de mouvement du sillage réel par la loi $u=-\theta S\left(s^{\prime}\right)$ où $\theta$ est un paramètre à déterminer et $S\left(s^{\prime}\right)$ est le taux d'extinction du défaut de vitesse vers l'aval ( $s$ désigne l'abscisse d'un point du sillage comptée à partir de l'arrière de la cavité ; $0<S\left(s^{\prime}\right) \leqslant 1$ ). Suivant les quelques indications expérimentales disponibles et aussi en raison des contraintes de l'analyse, on a choisi pour le taux d'extinction la loi : $S\left(s^{\prime}\right)=\exp \left(-s^{\prime} / \ell\right)$, la longueur de la cavité étant, plus que la corde du profil, la grandeur significative dans cette partie de l'écoulement (modelé appelé $S_{1}$ ).

La technique de résolution du problème mixte aux frontières ainsi posé consiste à représenter le domaine découlement sur un demi-plan auxiliaire $t$ - d'où la correspondance $z(t)$ - et, pour une longueur donnée de la cavité, de calculer la vitesse complexe de perturbation $w$ en fonction de $t$ par des expressions intégrales classiques (méthode de Signorini ou de Cheng et Rott par exemple). Quelques conditions de comportement doivent être aussi imposées à $w$ : nullité à l'infini amont $A$ et aval $G$ (fig. 4) ; continuité aux bords de fuite $D$ et $E$; singularité intégrable au bord d'attaque $: w \simeq z^{-1 / 2}$; vitesse transversale nulle dans le jet supérieur $B$. Dans le cas du modèle $S_{1}$, il faut formuler une condition supplémentaire afin de déterminer le paramètre de sillage $\theta$ : on a choisi de fixer l'épaisseur du sillage à l'infini aval, de manière à obtenir une configuration assez réaliste de l'écoulement ; en l'absence de données expérimentales suffisantes, on a imposé une valeur nulle de cette épaisseur.

Les calculs ont été faits dans le cas d'un coin symétrique de demi-angle $\gamma$ pour lequel on disposait d'informations détaillées sur la géométrie de la cavité. Il convient d'indiquer qu'en plus des difficultés classiques de ce genre de calcul (évaluation d'intégrales singulières -- distorsions introduites par la transformation conforme), l'introduction des conditions de survitesse non nulle dans le sillage de la cavité accroit la taille du problème d'un ordre de grandeur. Cependant la méthode choisie semble la plus adaptée pour traiter d'une manière précise les écoulements à cavités autour des profils considérés dont le rayon de courbure est inférieur à $2 \times 10^{-3}$

Nous donnons ici quelques résultats particulièrement significatifs, renvoyant pour plus de détails à la référence [7].

a) La relation $\ell(\sigma)$ est améliorée par le modèle $S_{1}$ puisque, à $\sigma$ donné, la longueur de la cavité est multipliée par un coefficient de l'ordre de 1,7 à 1,8 cepen- 


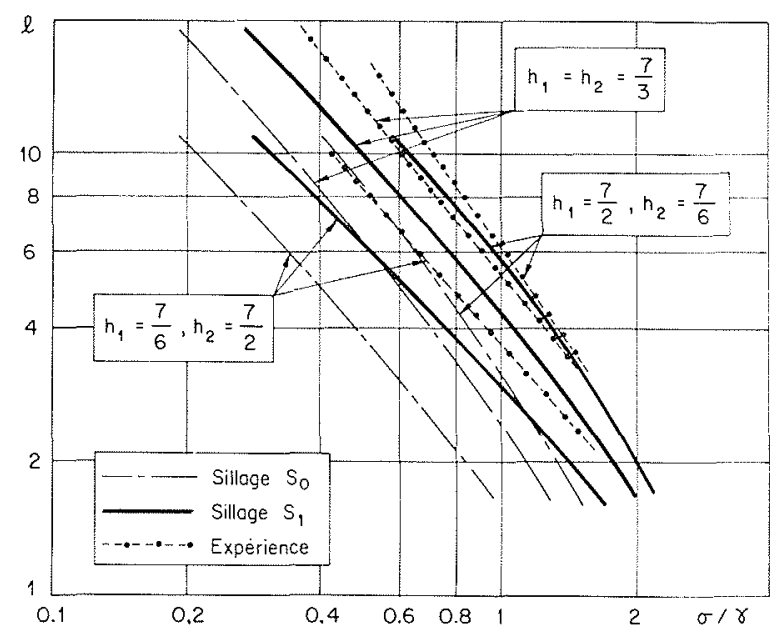

Figure 5 - Longueur de la cavité en fonction de $\sigma$ : comparaison avec l'expérience. Hauteur totale du canal : $h_{1}+h_{2}=14 / 3$. Incidence moyenne nulle.

dant que l'exposant $n$ de la loi expérimentale $\left(c^{\prime}\right)$ est à peu près retrouvé, comme dans le modèle $S_{0}$. (fig. 5).

b) Pour une longueur donnée de la cavité, la forme de la cavité est bien restituée par la théorie, au moins en ce qui concerne la frontière supérieure de la cavité, alors que le modèle $S_{0}$ donne une cavité nettement trop large.

c) Les coefficients de trainée $C_{x}$ déduits des deux modèles sont différents, à longueur de cavité égale, même pour les grandes longueurs de cavité, le coefficient de traînée donné par le sillage $S_{1}$ étant naturellement plus grand. Mais à dépression relative $\sigma$ égale, compte tenu des relations $\ell(\sigma)$ différentes, les coefficients de trainée coïncident presque parfaitement. L'accord entre la théorie et l'expérience concernant la relation $C_{x}(\sigma)$ n'est donc pas un critère assez sensible de validité de la théorie.

d) Les coefficients de portance $C_{z}$ donnés par les deux modèles diffèrent, soit à longueur donnée, soit même à nombre $\sigma$ donné, sauf pour les très petites valeurs du nombre $\sigma$. Cette différence est due presque entièrement à la différence de calage de l'incidence d'adaptation et devient plus importante si le profil fonctionne au voisinage de la surface libre du canal.

On constate aussi que le paramètre de sillage $\theta$ se trouve très peu dépendant de la configuration géométrique adoptée : profondeur du canal, immersion et incidence du profil. Ce fait paraít favorable puisque, au facteur $2 \varepsilon$ près, $\theta$ représente le coefficient de pression à l'arrière de la cavité, coefficient qu'on peut prévoir fixé par les phénomènes de dissipation localisés dans cette région, liés à la dynamique interne de l'écoulement.

\section{Influence de la gravité}

L'importance du rôle joué par la surface libre et la cavité dans le comportement du profil laisse prévoir une influence non négligeable des effets de la gravité. Une telle influence a été reconnue comme faible dans le cas d'un profil supercavitant à extrados dénoyé fonctionnant en milieu infini [8]. Nous avons observé au contraire, au cours des divers essais effectués en tunnel à des vitesses différentes, que le coefficient de portance d'un profil ventilé de $8 \mathrm{~cm}$ de corde, placé à la profondeur d'immersion d'une corde, peut varier d'environ $20 \%$ lorsque la vitesse de l'écoulement amont passe de $4 \mathrm{~m} / \mathrm{s}$ à $10 \mathrm{~m} / \mathrm{s}$ (fig. 6). En fait, cette variation du coefficient de portance est d'autant plus grande que le nombre de ventilation est plus petit. Elle s'accompagne également d'une modification de la loi $\ell(\sigma)$ suivant la vitesse de l'écoulement (fig. 7). Il n'existe malheureusement pas d'étude applicable directement au cas qui nous intéresse qui permette de conclure que les variations observées sont bien liées à une influence de la gravité. En raison de son importance, nous nous attachons actuellement à résoudre ce problème sur le plan théorique. Ce qui est en cause est évidemment d'une part l'interprétation des essais effectués en laboratoire à vitesse réduite, essais pour lesquels le nombre de

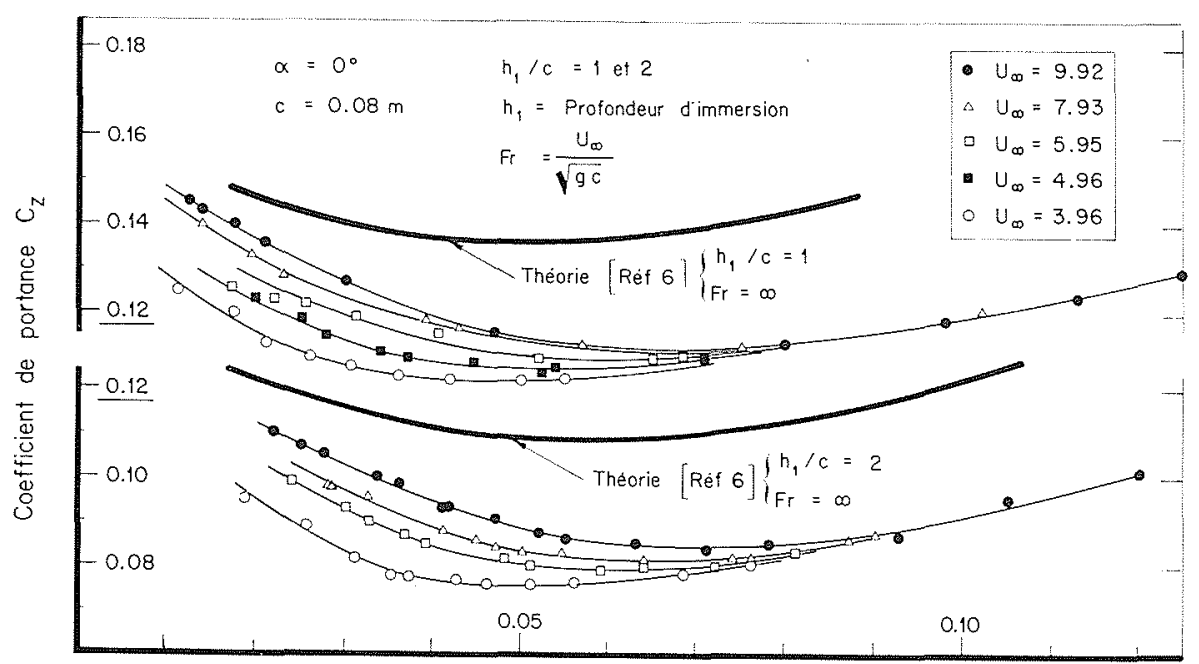

Nombre de ventilation $\sigma_{\exp }=\left(P_{\infty}-P_{c}\right) / \frac{1}{2} p \cup_{\infty}^{2}$

Figure $6-C_{z}(\sigma)$ expérimental. 


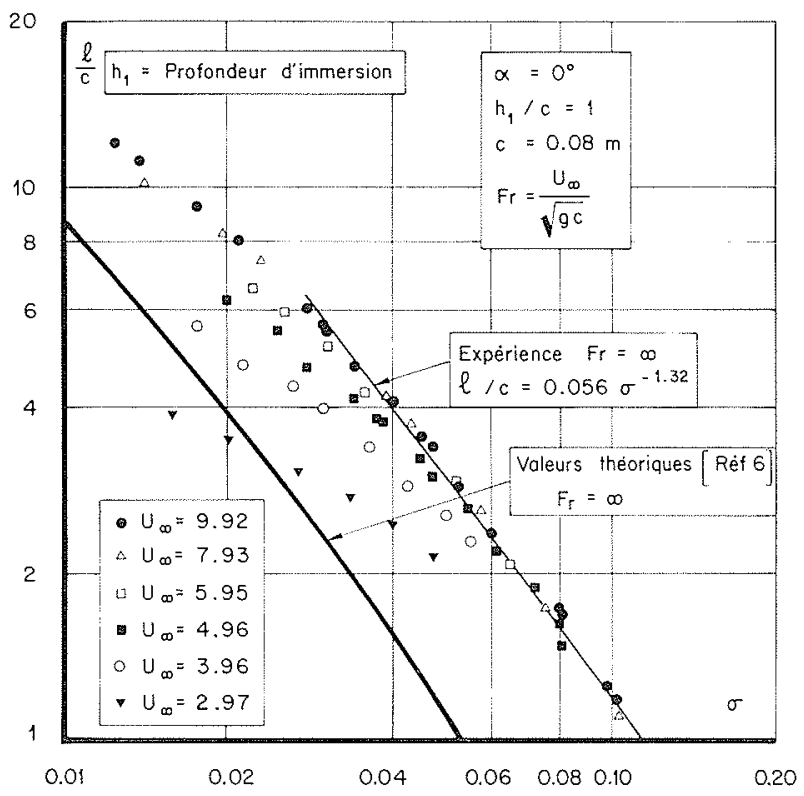

Figure 7 - Influence de la vitesse sur la longueur de la cavité.

Froude n'est pas toujours grand, d'autre part la prévision correcte de la portance d'une aile réelle. On doit en effet observer que dans la définition du nombre de Froude à prendre en compte pour l'estimation des effets de la gravité, la longueur caractéristique à retenir est celle de la cavité, qui est grande vis-à-vis de la longueur de la corde du profil. Pour des longueurs de cavité de l'ordre d'une dizaine de cordes, le nombre de Froude ainsi défini reste compris entre 1 et 3 , pour une aile de $1,20 \mathrm{nn}$ de corde se déplaçant à des vitesses comprises entre 22 et 66 nœuds. On atteint ainsi des valeurs du nombre de Froude pour lesquelles la théorie classique démontre une influence significative de la gravité [9], dans le cas plus simple des profils de type subcavitant. Le développement des calculs en cours ne permet pas encore de conclure si l'écart de $20 \%$ observé peut être imputable uniquement a cette influence. Le modèle utilisé pour l'étudier est celui du paragraphe 3 ; on emploie une méthode de perturbation traitée de la même manière que celle qu'on utilise pour un écoulement de fluide non pesant. Comme il a été exposé, les difficultés de calcul numérique sont considérables ; on peut néanmoins résoudre le problème avec précision en étudiant analytiquement le comportement asymptotique de la surface libre à l'infini.

\section{Comportement d'ailes d'envergure finie}

Même menée à son terme, l'étude de l'écoulement autour $d$ un profil d'aile bidimensionnel, tenant compte de toutes les causes possibles de perturbation verticale, n'est pas suffisante pour prévoir avec précision les caractéristiques d'une aile réelle d'envergure finie. L'étude de l'écoulement tridimensionnel pose certains problèmes concernant la validité des schémas de calcul retenus pour représenter cet écoulement, notamment dans la zone de fermeture de la cavité et à l'extrémité de l'aile. Pour cette raison, depuis un an, nous avons effectué un grand nombre d'essais en vue de recueillir des informations concernant l'organisation des nappes tourbillonnaires qui s'échappent d'une aile tridimensionnelle. On observe en général la formation de deux cavités : une cavité médiane qui s'étend sur toute la longueur de l'envergure et une cavité marginale qui prend naissance à l'extrémité du bord de fuite de l'aile et qui s'identifie au tourbillon marginal. Lorsque l'incidence du profil varie, le débit d'air étant maintenu constant, les longueurs respectives de la cavité médiane et de la cavité marginale varient simultanément. Lorsque l'incidence devient grande, la cavité médiane se raccourcit alors que la cavité marginale devient de plus en plus importante. A partir d'un certain angle limite qui dépend de la vitesse de l'écoulement, la cavité marginale vient s'accrocher à l'extrémité du bord d'attaque de l'aile et recouvre une partie de l'extrados. A ce moment, on n'observe pas de cavitation sur l'extrados de l'aile et si l'angle d'incidence augmente encore, on obtient le décrochage de l'aile sans qu'il y ait eu apparition de cavitation. Ce type de comportement est valable pour une demi-aile de forme en plan carrée (allongement équivalent à 2) obtenue à partir d'un profil d'aile optimisé vis-à-vis des risques de cavitation [6]. Les résultats quantitatifs obtenus pour cette même aile autorisent des espoirs raisonnables concernant la possibilité d'utilisation d'une aile ventilée pour un hydroptère rapide : il s'avère que les effets de déflection qui ont pour conséquence de diminuer la portance, et aussi la pente $d C_{z} / d \alpha$, favorisent l'obtention d'une grande fourchette de non-cavitation. Ainsi pour l'aile de forme en plan dont il est question, la fourchette de non-cavitation pour un nombre de cavitation $\sigma_{v}$ égal à 0,19 atteint la valeur $\Delta \alpha \simeq 6^{\circ}$. Le domaine de stabilité de l'aile est naturellement augmenté d'autant, sans que la finesse soit trop pénalisée par rapport au profil bidimensionnel : elle reste comprise entre 8 et 9 (valeurs prises au milieu de la fourchette de non-cavitation) pour une dépression relative $\sigma$ variant entre 0,04 et 0,02 . En écoulement bidimensionnel, on obtiendrait la valeur 14 à $\sigma=0,025$. La pente $d C_{z} / d \alpha$ devient égale à environ $2,5 \mathrm{rad}^{-1}$; cette valeur correspond assez bien à la valeur obtenue en écoulement bi-dimensionnel $\left(5,25 \mathrm{rad}^{-1}\right)$ corrigée par la théorie de la ligne portante. Cela explique pour la plus grande part le fait que la fourchette de noncavitation soit multipliée par un facteur trois. Il semblerait que les effets tridimensionnels, accentués par le choix d'un allongement réduit et par la présence de la cavité marginale qui, en dehors des conditions d'adaptation, devient très importante, aient pour conséquence de lisser la courbe de pression longitudinale de l'extrados qui, en écoulement bi-dimensionnel, présente un gradient très accentué au voisinage du bord d'attaque. Si cela était vérifié, l'utilisation d'ailes à corde peu évolutive et extrémité droite serait préférable. De plus, à grande vitesse, le passage au régime supercavitant pur par augmentation de l'incidence ne s'accompagne pas d'une grande diminution du coefficient de portance. Pratiquement, ce coefficient évolue de manière à peu près continue avec néanmoins des petites discontinuités observées au cours de la transition : ces discontinuités sont liées à la formation de cones de superventilation qui prennent naissance au bord d'attaque du profil. Il ne paraît pas impossible de contróler la forma- 
tion de ces cones de manière à régulariser la courbe de portance si cela s'avère nécessaire.

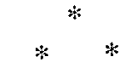

Les résultats présentés dans cet article montrent l'intérêt des profils supercavitants à extrados noyé. Signalons aussi que ces profils sont bien adaptés à la définition des géométries mixtes nécessaires pour obtenir de grands écarts de vitesse (par exemple : envol à 35 nœuds, vitesse de croisière économique à 45 nœuds, vitesse maximale : 80 nœuds). La faisabilité d'un hydroptère muni de tels types d'ailes apparait alors toutà-fait accessible à condition que l'on dispose de la puissance propulsive nécessaire. Il semble même que les ailes à extrados noyé soient aussi bien adaptées à l'ensemble des spécifications requises que les ailes à extrados dénoyé dont les qualités pour le vol à grande vitesse ont déjà été décrites [10]. Les espoirs fondés sur ce type d'ailes demandent, pour étre concrétisés, des études approfondies dans différents domaines dont les principaux concernent la définition des formes en plan, le vrillage, la stabilité, les interactions et les effets instationnaires.

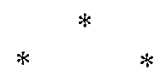

Cette étude a été financée par la Direction des Recherches et Moyens d'Essais (conventions $n^{\circ} 74.351$ et 75.297).

\section{Références}

[1] TULIN M.P. - Steady two-dimensional cavity flows about slender bodies. D.T.M.B. Rept 834 (1953).

[2] WU T.Y.T. - WANG D.P. - A wake model for free streamline theory. J. of Fluid Mech. 18 (1963), pp. 65-93.

[3] TULIN M.P. - Supercavitating flows. Small perturbation theory, J. of Ship Res. 8 (1964), pp. 16-37.

[4] DODU J., DUPORT J., MICHEL J.M. - Le tunnel hydrodynamique de l'Université de Grenoble. La Houille Blanche (1968), pp. 697-702.

[5] MICHEL J.M., ROWE A. - Profils minces supercavitants à arrière tronqué. La Houille Blanche, 1974. I. Définition et étude théorique de profils portants à nombre de ventilation nul en présence d'une surface libre. MICHEL J.M. et ROWE A. La H.B., 3 (1974), pp. 205-214. II. Influence de la ventilation sur les caractéristiques de profils portants évoluant au voisinage d'une surface libre. ROWE A. La H.B., 3 (1974), pp. 215-230. III. Etude physique du sillage en écoulement plan. MICHEL J.M. La H.B., 6 (1974), pp. 429-445

[6] ROWE A., MICHEL J.M. - Two-dimensional basevented hydrofoils near a free surface : Influence of the ventilation number. $J$. of Fluids Eng., 97, series $1, n^{\circ} 4$ (Déc. 1975), pp. 465-474.

[7] MICHEL J.M. - Wakes of developed cavities. $J$. of Ship Research (à paraître).

[8] LAROCK B.E., STREET R.L. - A non-linear theory for a full cavitating hydrofoil in a transverse gravity field. J. of Fluid Mech. 29, 2 (1967), pp. 317-366.

[9] PLOTKIN A. - The thin-hydrofoil thickness problem including leadingedge corrections. J. of Ship Res. 19, 2 (June 1975), pp. 122-129.

[10] WANG D.P., SHEN Y.T. - A validation study of the mixed-foil concept for high-speed hydrofoils., J. of Ship Res. 20, 2 (June 1976), pp. 85-94.

\title{
Discussion
}

\author{
Président: M. A. CASTERA
}

M. le Président remercie M. DODU de son exposé sur un sujet de grande actualité dans le domaine de la sustentation et de la propulsion des navires non conventionnels.

Comme l'Université de Grenoble, poursuit-il, le Bassin d'Essais des Carènes dispose d'un tunnel de cavitation à surface libre. Quelques difficultés y sont rencontrées pour maintenir suffisamment constantes les caractéristiques du milieu - et en particulier la teneur en air au cours d'une expérimentation prolongée. Ceci est lié à la grande surface libre de la cuve aval. Le problème se pose-t-il de façon aigue à Grenoble?

Je pense, dit M. DODU, qu'il n'est pas déraisonnable d'imaginer deux types d'essais assez différents suivant que la dépression relative joue ou non le rôle essentiel dans l'expérience effectuée.

Dans le premier cas -celui de la cavitation développée- on peut penser que les phénomènes fortement liés à la thermodynamique des échanges entre l'eau et l'air seront très estompés et il y a beaucoup de chances que l'on réalise, sans trop de difficultés, des essais reproductibles.

Par contre, si les phénomènes étudiés dépendent peu de la dépression relative mais beaucoup plus du paramètre de Thoma, la teneur en air du liquide jouera un rôle déterminant.

A l'Institut de Mécanique de Grenoble, nous avons fait des tentatives dans le sens qui répond à votre préoccupation, d'une part, en comparant divers procédés pour la mesure de la teneur en air et d'autre part, lors de la construction du tunnel, en prenant des précautions pour qu'il soit possible de faire varier la teneur en gaz, indépendamment des autres conditions, en particulier, des conditions de pression.

Peut-on établir un schéma entièrement cohérent -au point de vue de la Mécanique des Fluides- de la fermeture de la poche en régime permanent et en l'absence de viscosité ? demande M. EUVRARD.

En ce qui concerne la physique, répond M. DODU, nous ne disposons pas d'un schéma définitif expliquant totalement le déroulement du phénomène. Toutefois M. MICHEL (La Houille Blanche, $\mathrm{n}^{\circ}$ 6-1974, p. 429-445) a mis en lumière les aspects principaux des mécanismes de fermeture de la cavité. Quoi qu'il en soit, les schémas de type hydrodynamique, actuellement disponibles, n'incluent pas la dissipation visqueuse. Il s'ensuit une traînée globale nulle sur le profil et la représentation de la cavité sans que cette traînée soit nulle sur le profil seul.

M. Le Président remercie M. DODU et ses collaborateurs et donne la parole à M. BAUBEAU pour l'exposé de la Communication qu'il a établie en collaboration avec M. BALQUET. 


\begin{tabular}{|c|c|}
\hline \multicolumn{2}{|c|}{ Abstract } \\
\hline $\begin{array}{l}\text { Increasing requirements for high-speed navigation have been } \\
\text { focussing interest on such designs as hydrofoils for which cavi- } \\
\text { tation or ventilation are acceptable in that they do not result } \\
\text { in an excessive reduction in aspect ratio. } \\
\text { Results of present experimental and theoretical research on } \\
\text { base-ventilated hydrofoils at the Grenoble Institute of Fluid } \\
\text { Mechanics are presented. Investigation of hydrofoil behaviour } \\
\text { in two-dimensional flow is considered to be a good starting- } \\
\text { point, as it lends itself to more convenient experimental inves- } \\
\text { tigation and more rewarding analytical model simulation, e.g. } \\
\text { conformal representation, linearisation or connected asymp- } \\
\text { totic expansion. Comparative reference is made to the fre- } \\
\text { quently significant effect of prototype hydrofoil behaviour } \\
\text { which, in the case of thin hydrofoils causing minor deviation } \\
\text { of the initially undisturbed flow, is invariably marked by a } \\
\text { lack of symmetry (effect of surface and bed boundaries, } \\
\text { gravity or limited span). } \\
\text { Main flow-governing parameters include hydrofoil and } \\
\text { flow channel geometry, physical fluid properties, gravity, } \\
\text { velocity at infinity and boundary pressures. Thus, experi- } \\
\text { mental and theoretical investigations come under two cate- } \\
\text { gories, one relating to overall cavity geometry, forces and } \\
\text { general properties (for which the non-dimensional difference } \\
\text { between the reference pressure at infinity and cavity pressure } \\
\text { is most significant), and the other in which cavity pressure is } \\
\text { investigated as a parameter responding to initial data (mainly } \\
\text { ventilation flow). A satisfactory explanation has been found } \\
\text { for the first case, but the second will require further research } \\
\text { to elucidate the air entrainment processes for which the geo- }\end{array}$ & $\begin{array}{l}\text { metrical and dynamic "micro-structure" of the flow appears } \\
\text { to be significant. } \\
\text { Recent experimental and theoretical lift research has shown } \\
\text { the effect of a free surface on incidence to determine a distinct } \\
\text { minimum on the lift vs. relative depression characteristic } \\
\text { (Fig. 2). In addition, the theoretical data show calculated lift } \\
\text { to depend very closely on cavity geometry, and therefore, the } \\
\text { need for a more "refined" analytical model. } \\
\text { Marked model simulation progress has been made by } \\
\text { allowing for existing momentum deficiency after the cavity } \\
\text { ("negative overspeed" in the wake). With a model improved } \\
\text { to allow for this factor, cavity geometry and variation with } \\
\text { relative depression can be determined more exactly, and } \\
\text { therefore, more realistic simulation of specific hydrodynamic } \\
\text { properties achieved. } \\
\text { An analytical study of experimentally-confirmed gravity } \\
\text { effect (fig. } 6 \text { and } 7 \text { ) on a first-order perturbation model (now } \\
\text { in progress), is expected to provide an estimated order of } \\
\text { magnitude for this factor. } \\
\text { An experimental study of three-dimensional flow past } \\
\text { hydrofoils of finite span has provided extensive data for use } \\
\text { in establishing appropriate theoretical schemes. Though the } \\
\text { existence of variegated forms of cavity (median and marginal } \\
\text { cavities associated with circulation around the hydrofoil } \\
\text { span) singularly complicates flow description, some general } \\
\text { data have emerged, e.g. reduction of the lift/incidence ratio } \\
\text { (dC } C_{2} / d x \text { ), correlative non-cavitating leading-edge ranges tend- } \\
\text { ing to improve hydrofoil stability. }\end{array}$ \\
\hline
\end{tabular}

